\title{
Middle and long-term outcomes of non-ring tricuspid annuloplasty in patients with mitral valve replacement
}

\section{Yongjun Qian ( $\nabla$ qianyongjun@scu.edu.cn )}

Sichuan University West China Hospital https://orcid.org/0000-0002-8013-4070

\section{Dou Yuan}

west china hospital of sichuan university

Liping Chen

west china hospital of sichuan university

Tao Li

west china hospital of sichuan university

Yunfei Ling

wes china hospital of sichuan university

Xijun Xiao

west china hospital of sichuan university

\section{Research article}

Keywords: Mitral valve replacement, non-ring tricuspid annuloplasty, long-term observation

Posted Date: December 31st, 2020

DOI: https://doi.org/10.21203/rs.3.rs-136778/v1

License: () (1) This work is licensed under a Creative Commons Attribution 4.0 International License. Read Full License 


\section{Abstract \\ Objective}

To evaluate the middle-period and long-term outcomes of one-stage non-ring TVP in patients with MVR.

\section{Methods}

427 patients who received one-stage mechanical MVR and non-ring TVP from January 2005 to January 2009 at Department of Cardiovascular Surgery of West China Hospital; After screening, 400 patients were finally recruited, who reexamined by UCG 10 years after surgery.and recorded UCG data.

\section{Results}

The patients were followed up for 10 years after surgery. Compared with the situation before surgery, $\mathrm{RV}(\mathrm{mm}): 21.47 \pm 3.14$ vs. $22.37 \pm 3.28, \operatorname{TAEDD}(\mathrm{mm}): 32.18 \pm 4.94$ vs. $35.88 \pm 4.07$ and $\operatorname{TESD}(\mathrm{mm}): 25.90 \pm 4.90$ vs. $28.12 \pm 4.12$ decreased significantly at 5 years after surgery $(P<0.05)$. However, RA(mm):49.35 \pm 13.05 vs. $50.69 \pm 12.90$ did not change significantly $(P>0.05)$. LVEF $(\%): 61.54$ \pm 8.23 vs. $57.38 \pm 8.87$ also increased significantly after surgery $(P<0.05)$. Compared with the preoperative situation, at 10 years after surgery, $\mathrm{RA}(\mathrm{mm})$ was $56.90 \pm 12.90, \mathrm{RV}(\mathrm{mm}) 23.12 \pm 4.58$, TESD (mm) $28.80 \pm 5.14$ and LVEF (\%) $59.81 \pm 8.95$, all of which increased significantly $(P<0.05)$; however, TEDD $(\mathrm{mm})$ was $35.41 \pm 5.11$, which did not change significantly $(P>0.05)$. Compared with preoperative situation, the ratios of severity of TR at 5 and 10 years after surgery improved significantly $(P<0.05)$.

\section{Conclusion}

It is reasonable to use the tricuspid annular diameter in patients with mitral valve disease as an indication for one-stage TVP. The one-stage non-ring TVP is conducive to preventing aggravation of TR, so as to achieve a better long-term outcome. Besides, oral diuretics are recommended to treat TR after surgery for such patients.

\section{Introduction}

Mitral valve disease is the most common type of valvular heart disease, and about $50 \%-60 \%$ of the patients with mitral valve disease are combined with tricuspid regurgitation (TR) [1]. Moreover, residual or aggravating TR after mitral valve replacement(MVR) is not conducive to the short-term recovery and longterm survival of the patients. Therefore, TR remains a main challenge for cardiac surgeons [2, 3]. Whether to treat TR simultaneously as MVR and the timing and approach to treat are still a topic of controversy. So far, European Society of Cardiology (ESC) [4] and American College of Cardiology/American Heart Association (ACC/AHA) [5] seem to share similar opinions on the treatment of severe TR in MVR. 
However, the opinions diverge on whether to treat mild tricuspid regurgitation simultaneously. There are also conflicting views on which surgical approach is better for tricuspid annuloplasty(TVP) [6, 7].

In the present study, tricuspid annular diameter was considered as an indication for one-stage TVP. Moreover, the one-stage non-ring TVP was performed. This study reported the long-term outcome of such a treatment strategy for the patients.

\section{Methods}

\section{Clinical data}

A total of 427 patients who received one-stage mechanical MVR and TVP from January 2005 to January 2009 at Department of Cardiovascular Surgery of West China Hospital; Within 10 years, 8 patients died , 7 patients were reoperated for severe TR, and 12 patients were reoperated for mitral valve problems. 400 patients were finally recruited, who reexamined by UCG『Ultrasonic Cardiogram $₫ 10$ years after surgery. After screening, A total of 400 patients who completed 10 years of follow-up were included in the study.The UCG datas of 400 patients were analyzed retrospectively. A total of 400 patients including 91

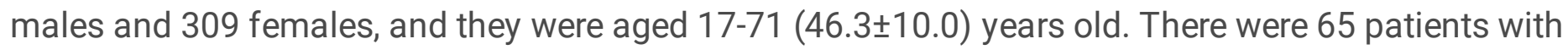
normal sinus heart rate, 52 patients with atrial flutter, and 283 patients with atrial fibrillation. According to the NYHA classification, there were 19 cases of class $\Downarrow$ cardiac function, 289 cases of class $\otimes$ cardiac function and 92 cases of class $\otimes$ cardiac function.

\section{Definition and classification of TR [8].}

TR was defined based on the ratio of regurgitation beam area and right atrial area upon UCG as well as the width of regurgitation beam. TR severity was graded on a $0-5$ scale: 0 , no TR; 1 , mild TR; 2 , mild to moderate TR; 3 , moderate TR; 4 , moderate to severe TR; 5 , severe TR.

\section{Indications for TVP}

(1) tricuspid annular dilation

Tricuspid annular diameter $\geq 40 \mathrm{~mm}$ or tricuspid annular diameter/body surface area $\geq 21 \mathrm{~mm} / \mathrm{m}^{2}$, upon intraoperative transesophageal UCG; distance from the junction between anterior and septal leaflets to the junction between anterior and posterior leaflets $\geq 70 \mathrm{~mm}$; (2) no tricuspid annular dilation, but with apparent TR

No apparent tricuspid annular dilation found by preoperative or intraoperative ultrasound, but $T R \geq 1$ revealed by perioperative ultrasound.

\section{Surgical procedures}


The patients took a supine position and received total intravenous anesthesia with endotracheal intubation for assisted ventilation. Full sternotomy was performed, and extracorporeal circulation was established. Myocardial protection was given by moderate hypothermia and hypothermic cardioplegia perfusion. Mitral valve and tricuspid valve surgeries were performed via the right atrium-interauricular septum pathway. After mechanical MVR and other intracardiac procedures were over, tricuspid annular dilation and leaflet alignment were examined by water injection. For patients without tricuspid annular dilation but severe TR (all 8 patients belonged to mild to moderate TR or moderate TR), 5-0 prolene suture was used for intermittent suture of leaflet defect (anterior leaflet). For 298 patients with tricuspid annular dilation and leaflet defect, leaflet repair was first performed (anterior leaflet, and junction between anterior leaflet and septal leaflet or septal leaflet also included for a few patients), which was followed by onestage non-ring TVP: one-stage bicuspidization (2-0 double-headed MVR thread was led in from the junction between anterior and posterior leaflets and led out from the junction between the posterior and septal leaflets after interrupted suture along the valve annulus; the thread was knotted and immobilized after padding) and modified Kay's annuloplasty [9] (2-0 double-headed MVR thread was led in vertically from the junction between anterior and posterior leaflets and led out from within the valve annulus; then the needle was inserted into the valve annulus from the junction between the posterior and septal leaflets and led out from outside the valve annulus. The thread was knotted and immobilized after padding). Thus, TVP was finished. For those without leaflet defect, one-stage non-ring TVP was directly performed. Following TVP, the size of valve opening was inspected with fingers, and leaflet alignment and TR were

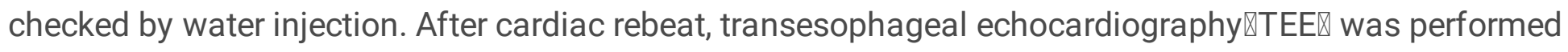
again to assess the tricuspid valve. After surgery, the patients were sent to ICU for continuous monitoring and supportive treatment.

\section{Follow-up}

Patients' gender, age, heart rate, cardiac function, date and methods of surgery were recorded before discharge along with UCG data. UCG indicators measured were as follows: Left atrium diameter (LA), left ventricle diameter (LV), right atrium diameter (RA), right ventricle diameter (RA), left ventricular ejection fraction (LVEF), tricuspid end-diastolic diameter (TEDD), tricuspid end-systolic diameter (TESD) and tricuspid regurgitation (TR). After discharge, the patients were required to receive regular follow-up at the outpatient clinic and reexamination by UCG.

\section{Statistical}

All statistical analyses were performed using SPSS 19.0 software. Measurements were expressed as mean \pm standard deviation $(X(-) \pm S)$, and paired t-test was used for intergroup comparisons. Counts were compared between the groups by using the chi-square test. $P \leq 0.05$ indicated significant difference.

\section{Results}

The patients were followed up for 10 years. At 5 years after surgery, RA, LA and LV did not change significantly compared with the preoperative values $(P>0.05)$; however, RV, TEDD and TESD decreased 
significantly, while EF increased significantly $(P<0.05)$. At 10 years after surgery, TEDD did not change significantly compared with the preoperative values $(P>0.05)$; however, RA, RV, LA, LV, TESD and EF increased significantly $(P<0.05)$. Compared with the values at 5 years after surgery, RA, RV, LA, LV, TEDD and TESD at 10 years after surgery increased significantly, while EF decreased significantly $(P<0.05)$. (Table)

The ratios of severity of TR at 5 and 10 years after surgery were considerably superior to those before surgery $(P<0.05)$. There was an apparent increase in the proportion of patients with no or mild TR, while the proportion of patients with TR of moderate or above severity decreased dramatically.(Figure )

\section{Discussion}

In spite of the remarkable progress in the diagnostic and treatment techniques for valvular heart diseases, TR may still appear or even aggravate after MVR as time goes. Even if no apparent TR is observed by intraoperative examination, patients receiving left heart valve surgery are still faced with the risk of TR in the long term [10].

There are conflicting opinions and clinical practice concerning whether TVP should be performed simultaneously with MVR. Such divergence is not only related to the use of different guidelines, but also reflects varying cognition about this issue among cardiac surgeons from different regions or countries. At present, only $7 \%-10 \%$ of the patients receive one-stage TVP at Mayo Clinic compared to as high as $65 \%$ at Mount Sinai Hospital [14].

Tricuspid valve has a complex structure, which consists of three leaflets (anterior, posterior and septal), chordae tendineae, musculi papillares, tricuspid annulus, right ventricle and right ventricular muscle [3]. The tricuspid annulus is an asymmetrical saddle-shaped structure, and effective leaflet alignment requires coordination between the valve ring, leaflet, chordae tendineae, musculi papillares and right heart function [7]. If any of them has problems, there will be hemodynamic changes leading to TR $[15,16]$.

It has been recognized in recent years that tricuspid annular dilation is an important mechanism involved in TR: Long-term right heart overload leads to right ventricular dilation, which further results in tricuspid annular dilation and TR [17]. In addition to tricuspid annular dilation, preload, afterload and right heart function are decisive factors related to the occurrence of TR[18-22]. Under different situations, these 3 factors can dramatically influence TR measurement. Quantitative evaluation of right heart function is of high importance for severity evaluation, decision making, efficacy and prognostic prediction, but few studies have been conducted in this respect [23]. That is an important cause of difficulty in an accurate evaluation of TR. The degree of tricuspid annular dilation is a more reliable and more constant indicator of pathological changes of the tricuspid valve $[6,22]$. Tricuspid annular dilation can be considered if any of the following three conditions are present: (1) TVAEDD/BSA $>21 \mathrm{~mm} / \mathrm{m}^{2}$ in the parasternal short axis view or apical four chamber view [24]; (2) TVAEDD $\geq 40 \mathrm{~mm}$ in any view [7, 19, 25]; (3) the length of line connecting the junction between the anterior and septal leaflets and the junction between the anterior and 
posterior leaflets $\geq 70 \mathrm{~mm}$ [22]. As was pointed out by Dreyfus, surgical treatment of mitral valve disease can only lower the right ventriclar afterload, but does not address tricuspid annular dilation and the impact on preload or right ventricular function. One-stage TVP should be considered for mitral valve disease with tricuspid annular dilation even without TR [22].

Since 2005, we began to use tricuspid annular diameter as a surgical indication for one-stage TVP. Follow-up results of the present study indicated at 5 years after surgery, the right ventricular diameter and tricuspid annular diameter decreased significantly, while the left heart function was improved considerably; moreover, the ratio of severity of TR was also improved. After the treatment, the proportion of patients with no or mild TR increased, while that of patients with TR of moderate or above severity decreased. We believe that it is more appropriate to use tricuspid annular dilation as an indication for one-stage TVP in patients receiving MVR instead of TR for the following reasons [14, 22]: (1) It has been pointed out that the tricuspid annular diameter is independently correlated with the severity of TR [26]; (2) The measurement of tricuspid annular dilation is relatively constant, while TR severity is more greatly influenced by preload, afterload and right heart function; (3) There may be inconsistencies between the clinical situation and hemodynamic indicators of patients; (4) Residual or aggravating TR after surgery adversely affects patients' recovery.

However, which surgical technique to use for TVP is another important issue where divergent opinions exist. Duran believed that partial ring shrinkage with any technique cannot withstand the continuous stress on the annulus in severe TR. Therefore, non-ring TVP can be used for mild to moderate TR, while ring TVP should be performed for severe TR [21].

Many researchers seem to suggest that when performing non-ring TVP, the efficacy is quite uncertain and there exists the risk of residual or aggravating TR after surgery in the long term $[2,7,13]$. But there are also opposing opinions holding that non-ring TVP is easier and more effective $[6,27,28]$. Ghanta et al. [20] compared the 3-year postoperative follow-up data of 237 cases receiving non-ring bicuspidization or ring TVP. The results showed that $25 \%$ of the patients receiving non-ring TVP suffered from moderate to severe TR, while $31 \%$ of the patients receiving TVP had moderate to severe TR. We believe that (1) ring TVP is also associated with the risk of recurrence of severe TR. Secondary surgery for these patients to remove the annuloplasty ring can avoid the occurrence of degree $\mathbb{Z}^{\circ}$ atrioventricular heart-block to some degree; (2) Most of the patients receiving left heart valve surgery came from remote regions, and onestage non-ring TVP was less costly, safer and easier for them. De Vega TVP was adopted at our hospital before 2003. During secondary surgery for those with postoperative severe TR, several patients were found with suture rupture near the middle part of anterior leaflet. Thus, De Vega TVP was abandoned for these patients. In this study, modified Kay's annuloplasty was chosen due to its lesser impact on the anterior leaflet and shorter suture thread after immobilization, which makes it less likely to rupture.

Therefore, one-stage TVP was expected to achieve similar long-term outcomes. The 10-year follow-up data indicated that there was a significant reduction in the left and right atrial and ventricular diameter and tricuspid annular diameter at end-systole, while the left heart function was improved dramatically. 
The ratio of severity of TR was also improved effectively, with much more patients having no or mild TR after surgery and no significant increase in the proportion of patients with moderate and above TR. However, the tricuspid annular diameter at end-diastole was comparable before and after surgery. The clinical features of patients recruited in this study could be summarized as follows: (1) Predominantly rheumatic heart disease; (2) having not sought medical care until late and mostly coming from remote regions. Based on the above conditions, oral diuretics were given to those with TR revealed by UCG at 6 months after surgery and beyond. This medication was justified by the following reasons: (1) TR is a persistent and continuously aggravating pathological process [18, 22, 29]; (2) diuretics can lower the severity of hemodynamic disorder by alleviating right ventricular dilation and right atrial pressure, and spironolactone is more suitable for patients with right heart failure [30].

\section{Conclusion}

It is reasonable to use the tricuspid annular diameter in patients with mitral valve disease as an indication for one-stage TVP. The one-stage non-ring TVP is conducive to preventing aggravation of TR, so as to achieve a better long-term outcome. Because,Most of the patients receiving left heart valve surgery came from remote regions in china, and one-stage non-ring TVP was less costly, safer and easier for them. Besides, oral diuretics are recommended to treat TR after surgery for such patients.

\section{Abbreviations}

TR, Tricuspid regurgitation; MVR, Mitral valve replacement; ESC, European Society of Cardiology; ACC/AHA ,American College of Cardiology/American Heart Association; TVP,tricuspid annuloplasty; UCG, Ultrasonic Cardiogram; TEE, transesophageal echocardiography; LA ,Left atrium diameter; LV, Left ventricle diameter; RA, Right atrium diameter; RA, Right ventricle diameter; LVEF, Left ventricular ejection fraction ;TEDD, Tricuspid end-diastolic diameter;TESD, Tricuspid end-systolic diameter .

\section{Declarations}

Acknowledgements: Not applicable

Ethics approval and consent to participateIThe study protocol was approved by the local ethics committee (Sichuan University West China Hospital) and the study was performed according to the Declaration of Helsinki.

Consent for publicationロAll co-authors permit editorial amendments and the paper's publication.

Availability of data and material: All the data and material were restored in the 
database of West China Hospital.

Competing interests: The authors declare that they have no conflict of interest.

Funding: This work was supported by grants from the National Key Project Program during the 13th 5Year Plan Period of China (2016YFC1302003), and from the Major Science and Technology Project of Sichuan Province, China (No. 2017SZ0056), and the Scientific Research Project of National Clinical Research Center for Geriatrics,West China Hospital, Sichuan University (No. Z2018B19).

Authors' contributions: Yongjun Qian and Xijun Xiao conceived and designed the study. Dou Yuan and Liping Chen wrote the paper. Tao Li, Yunfei Ling, Yongjun Qian reviewed and edited the manuscript. All authors read and approved the manuscript.

Consent to Publish: Written informed consent to publish personal or clinical details were obtained from all the study participants.

\section{References}

[1] Boyaci A, Gokce V, Topaloglu S, et al. Outcome of significant functional tricuspid regurgitation late aft er MVR for predominant rheumatic mitral stenosis. Angiology 2007;58(3):336-342.

[2] Kuwaki K, Morishita K, Tsukamoto M, et al. Tricuspid valve surgery for functional tricuspid valve regurgitation associated with left-sided valvular disease. Eur J Cardiothorac Surg 2001;20(2):577-582.

[3] Navia JL, Nowicki ER, Blackstone EH, et al. Surgical management of secondary tricuspid valve regurgitation: Annulus, commissure, or leaflet procedure?. J Thorac Cardiovasc Surg 2010;139(6): 14731482.

[4] Baumgartner H, Falk V, Bax JJ, et al. 2017 ESC/EACTS Guidelines for the Management of Valvular Heart Disease. Rev Esp Cardiol (Engl Ed)2018; 71(2):110.

[5] Nishimura RA, Otto CM, Bonow RO, et al.2017 AHA/ACC Focused Update of the 2014 AHA/ACC Guideline for the Management of Patients With Valvular Heart Disease: A Report of the American College of Cardiology/American Heart Association Task Force on Clinical Practice Guidelines. J Am Coll Cardiol 2017;70(2):252-289.

[6] Naqshloand MS , Abid AR, Akhtar RP, et al. Functional tricuspid regurgitation in rheumatic heart

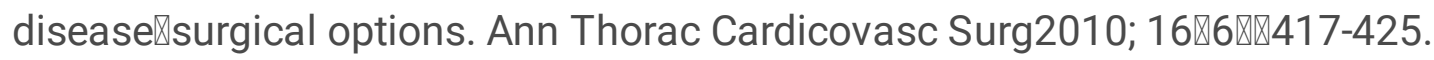

[7] Rogers JH, Bolling SF. Valve repair for functional tricuspid valve regurgitation囚anatomical and surgical considerations. Semin Thorac Cardiovasc Surg 2010;22ه18ख84-89. 
[8] Colombo T, Russo C, Ciliberto GR, et al. Tricuspid regurgitation secondary to mitral valve disease: tricuspid annulus function as guide to tricuspic valve repair. Cardiovasc Surg 2001;9(4): 369-377.

[9] Wang J, Li Z, Zhu Q, et al. A modified tricuspid valve annuloplasty technique for functional tricuspid regurgitation. Chin Med J (Eng I) 2013;126 (18):3534-3538.

[10]Tager R, Skudicky D, Mueller U, et al. Long-term follow-up of rheumatic patients undergoing left-sided valve replacement with tricuspid, annuloplasty-validity of preoperative echocardiographic criteria in the decision to perform tricuspid annuloplasty. Am J Cardiol 1998;81(8): 1013-1016.

[11] Nishimura RA, Otto CM, Bonow RO, et al. 2014 AHA/ACC Guideline for the management of patients with valvular heart disease: a report of the American College of Cardiology/American Heart Association Task Force on Practice Guidelines. Circulation 2014;129(2): e521-643.

[12]Vahanian A, Alfieri O, Andreotti F, et al.Guidelines on the management of valvular heart disease( version 2012): the Joint Task Force on the Management of Valvular Heart Disease of the European Society of Cardiology (ESC) and the European Association for Cardio-Thoracic Surgery (EACTS). Eur J Cardiothorac Surg 2012;42(4): S1-44.

[13] McCarthy PM, Sales VL. Evolving indications for tricuspid valve surgery. Curr Treat Options Cardiovasc Med 2010; 12(6): 587-597.

[14] Dion RA. Is the air in Toronto, Rochester, and Cleveland different from that in London, Monaco, Leiden, Genk, Milan, and New York? J Thorac Cardiovasc Surg2015; 150(5): 1040-1043.

[15] AHA; ACC; et al. AHA/ACC guidelines for secondary prevention for patients with coronary and other atherosclerotic vascular disease: 2006 update endorsed by the National Heart, Lung,and Blood Institute. J Am Coll Cardiol 2006;47(10): 2130-2139.

[16] Baumgartner H, Falk V, Bax JJ, et al. 2017 ESC/EACTS Guidelines for the management of valvular heart disease. Eur Heart J 2017; 38(36):2739-2791.

[17] Benedetto U, Melina G, Angeloni E, et al.)Prophylactic tricuspid annuloplasty in patients with dilated tricuspid annulus undergoing mitral valve surgery. J Th orac Cardiovasc Surg 2012; 143(3): 632-638.

[18] Bianchi G, Solinas M, Bevilacqua S, et al. Which patient undergoing mitral valve surgery should also have the tricuspid repair. Inter Cardiovasc Thorac Surg 2009; 9(6): 1009-1020.

[19] Bertrand PB, Koppers G, Verbrugge FH, et al. Tricuspid annuloplasty concomitant with mitral valve surgery: Effects on right ventricular remodeling. J Thorac Cardiovasc Surg 2014;147(4): 1256-1264.

[20] Ghanta RK, Chen R, Narayanasamy N, et al. Suture bicuspidization of the tricuspid valve versus ring annuloplasty for repair of functional tricuspid regurgitation: Midterm results of 237 consecutive patients. J Thorac Cardiovasc Surg 2007;133(1): 117-126. 
[21] Duran CM. Tricuspid valve surgery revisited. J Card Surg 1994;9(2 Suppl): 242-247.

[22] Dreyfus GD, Corbi PJ, Chan KMJ, et al. Secondary tricuspid regurgitation or dilatation: Which should be the criteria for surgical repair? Ann Thorac Surg 2005;79(1): 127-132.

[23] Rudski LG , Lai WW, Afilalo J, et al. Guidelines for the echocardiographic assessment of the right heart in adults: a report from the American Society of Echocardiography endorsed by the European Association of Echocardiography, a registered branch of the European Society of Cardiology, and the Canadian Society of

Echocardiography. J Am Soc Echocardiogr 2010;23(7): 685-713;quiz 786-788.

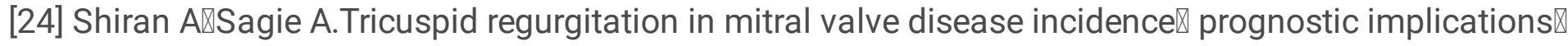
mechanism and management. J Am Coll Cardiol 2009; 53®5هष401-408

[25] Veire NRV, Braun J, Delgado V, et al. Tricuspid annuloplasty prevents right ventricular dilatation and progression of tricuspid regurgitation in patients with tricuspid annular dilatation undergoing mitral valve repair. J Th orac Cardiovasc Surg 2011;141(6):1431-1439.

[26] Park JH, Shin SH, Lee MJ, et al. Clinical and echocardiographic factors aff ecting tricuspid regurgitation severity in the patients with lone atrial fi brillation. J Cardiovasc Ultrasound 2015;23(3): 136142.

[27] Castedo E, Cañas A, Cabo RA, et al. Edge-to-edge tricuspid repair for redeveloped valve incompetence after DeVega's annuloplasty. Ann Thoracs Surg 2003;75(2): 605-606.

[28] Bernal JM, Gutiérrez-Morlote J, Llorca J, et al. Tricuspid valve repair: An old disease, a modern experience. Ann Thorac Surg 2004;78(6): 2069-2074.

[29]Chikwe J, Anyanwu AC. Surgical strategies for functional tricuspid regurgitation. Semi Thorac Cardivasc Surg 2010; 22(1): 90-96.

[30] Antunes MJ, Barlow JB. Management of tricuspid valve regurgitation . Heart 2007; 93(2): 271-276.

\section{Tables}

Table.1 Comparison of preoperative and postoperative cardiac chamber size, tricuspid annular diameter, left heart function and severity of TR 


\begin{tabular}{|c|c|c|c|}
\hline \multirow[t]{2}{*}{ UCG data } & Peroperative & 5 years postoperative & 10 years postoperative \\
\hline & $\bigotimes n=400 \rrbracket$ & $\bigotimes n=400 \rrbracket$ & $\bigotimes n=400 \rrbracket$ \\
\hline $\mathrm{RA} \otimes \mathrm{mm} \rrbracket$ & $50.69 \pm 12.90$ & $49.35 \pm 13.05$ & $56.90 \pm 12.90$ \\
\hline $\mathrm{RV} \rrbracket \mathrm{mm} \rrbracket$ & $22.37 \pm 3.28$ & $21.47 \pm 3.14$ & $23.12 \pm 4.58$ \\
\hline $\mathrm{LA} \rrbracket \mathrm{mm} \rrbracket$ & $54.18 \pm 14.68$ & $54.01 \pm 15.60$ & $59.66 \pm 19.30$ \\
\hline LV冈mm $\rrbracket$ & $47.46 \pm 6.74$ & $47.74 \pm 6.03$ & $49.44 \pm 8.44$ \\
\hline TEDD『mm $\rrbracket$ & $35.88 \pm 4.07$ & $32.18 \pm 4.94$ & $35.41 \pm 5.11$ \\
\hline TESD \mm $\rrbracket$ & $28.12 \pm 4.12$ & $25.90 \pm 4.90$ & $28.80 \pm 5.14$ \\
\hline LVEF囚\%区 & $57.38 \pm 8.87$ & $61.54 \pm 8.23$ & $59.81 \pm 8.95$ \\
\hline \multicolumn{4}{|l|}{ TRヌ\%】 } \\
\hline 0 & $20 \rrbracket 5.0 \rrbracket$ & 107囚26.7凶 & $29 \bigotimes 7.3 \rrbracket$ \\
\hline 1 & $182 \bigotimes 45.5 \rrbracket$ & 177ه44.3囚 & $146 \rrbracket 36.5 \rrbracket$ \\
\hline 2 & $76 \otimes 19.0 \otimes$ & $44 \otimes 11.0 \rrbracket$ & $115 \llbracket 28.8 \rrbracket$ \\
\hline 3 & 73ه18.3囚 & 53ه13.3ه & 67ه11.7ه \\
\hline 4 & 3187.7区 & $8 \otimes 2.0 \rrbracket$ & 24『6.0区 \\
\hline 5 & 18『4.5》 & 11区2.7区 & $19 \rrbracket 4.7 \rrbracket$ \\
\hline
\end{tabular}

Figures 


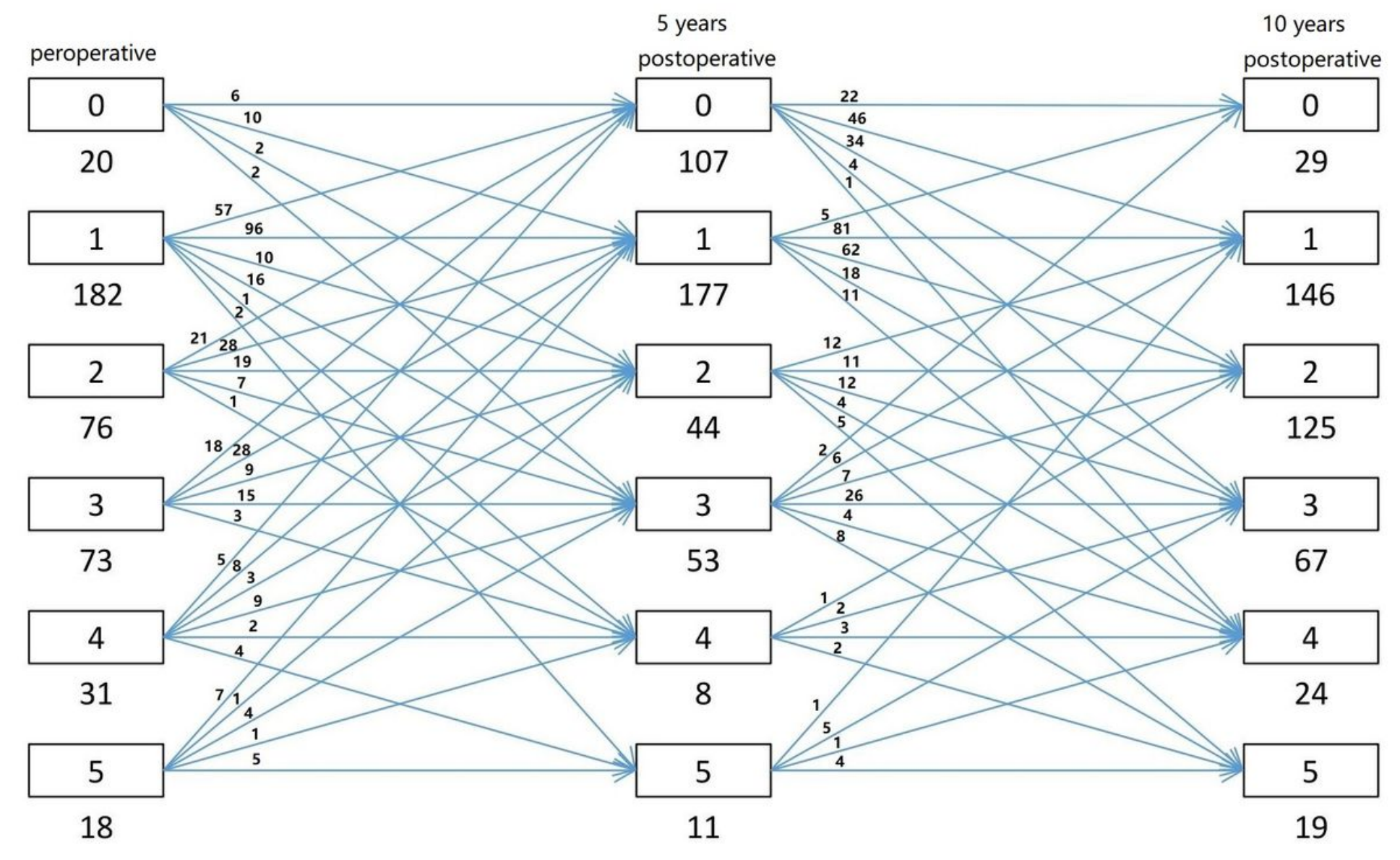

Figure 1

Changes of preoperative and postoperative severity of TR 